\title{
Stimulus Timing-Dependent Plasticity in Dorsal Cochlear Nucleus Is Altered in Tinnitus
}

\author{
Seth D. Koehler ${ }^{1,3}$ and Susan E. Shore ${ }^{1,2,3}$ \\ ${ }^{1}$ Kresge Hearing Research Institute, Department of Otolaryngology, University of Michigan Medical School, Ann Arbor, Michigan 48109, ${ }^{2}$ Department of \\ Molecular and Integrative Physiology, University of Michigan Medical School, Ann Arbor, Michigan 48109, and ${ }^{3}$ Department of Biomedical Engineering, \\ University of Michigan, Ann Arbor, Michigan 48105
}

Tinnitus and cochlear damage have been associated with changes in somatosensory-auditory integration and plasticity in the dorsal cochlear nucleus (DCN). Recently, we demonstrated in vivo that DCN bimodal plasticity is stimulus timing-dependent, with Hebbian and anti-Hebbian timing rules that reflect in vitro spike timing-dependent plasticity. In this in vivo study, we assessed the stimulus timing dependence of bimodal plasticity in a tinnitus model. Guinea pigs were exposed to a narrowband noise that produced a temporary elevation of auditory brainstem response thresholds. A total of $60 \%$ of the guinea pigs developed tinnitus as indicated by gap-induced prepulse inhibition of the acoustic startle. After noise exposure and tinnitus induction, stimulus timing-dependent plasticity was measured by comparing responses to sound before and after paired somatosensory and auditory stimulation presented with varying intervals and orders. In comparison with Sham and noise-exposed animals that did not develop tinnitus, timing rules in verified tinnitus animals were more likely to be anti-Hebbian and broader for those bimodal intervals in which the neural activity showed enhancement. Furthermore, units from exposed animals with tinnitus were more weakly suppressed than either Sham animals or exposed animals without tinnitus. The broadened timing rules in the enhancement phase in animals with tinnitus, and in the suppressive phase in exposed animals without tinnitus was in contrast to narrow, Hebbian-like timing rules in Sham animals. These findings implicate alterations in DCN bimodal spike timing-dependent plasticity as underlying mechanisms in tinnitus, opening the way for a therapeutic target.

Key words: tinnitus; bimodal; somatosensory; plasticity; cochlear damage; noise damage

\section{Introduction}

Tinnitus, the phantom perception of sound, has been linked to somatosensory innervation of the auditory system. Both tinnitus patients and normal subjects report that somatosensory stimuli, such as pressure on the face, can elicit or modulate tinnitus (Pinchoff et al., 1998; Levine, 1999; Sanchez et al., 2007). Converging somatosensory and auditory inputs are integrated in the dorsal cochlear nucleus (DCN), the first auditory brainstem nucleus (Kanold and Young, 2001; Haenggeli et al., 2005; Shore, 2005; Zhou et al., 2007; Kanold et al., 2011; Koehler et al., 2011) and a potential site for the initiation of tinnitus (Kaltenbach and McCaslin, 1996; Brozoski et al., 2002; Kaltenbach et al., 2004; Dehmel et al., 2012b).

DCN principal neurons (fusiform and giant cells) respond to somatosensory stimulation with excitatory and inhibitory responses lasting tens of milliseconds (Young et al., 1995; Davis et al., 1996; Kanold and Young, 2001; Shore, 2005) or minutes

Received June 21, 2013; revised Nov. 1, 2013; accepted Nov. 8, 2013.

Author contributions: S.D.K. and S.E.S. designed research; S.D.K. performed research; S.D.K. and S.E.S. contributed unpublished reagents/analytic tools; S.D.K. and S.E.S. analyzed data; S.D.K. and S.E.S. wrote the paper.

This work was supported by National Institutes of Health Grants R01 DC004825 and P30 DC05188. We thank Sanford Bledsoe, Roxana Stefanescu, David Martel, and Gregory Basura for their insightful comments; and David Martel, Malav Parikh, Yazan Kerallah, Krista Solem, and Ryan Richmond for providing invaluable assistance with data analysis.

The authors declare no competing financial interests.

Correspondence should be addressed to Dr. Susan E. Shore, 5434 Medical Science I, 1150 West Medical Center Drive, Ann Arbor, MI 48109. E-mail: sushore@umich.edu.

DOI:10.1523/JNEUROSCI.2788-13.2013

Copyright $\odot 2013$ the authors $\quad 0270-6474 / 13 / 3319647-10 \$ 15.00 / 0$
(Zhang and Guan, 2008). Fusiform cells "integrate" auditory and somatosensory stimuli presented in close temporal proximity by enhancing or suppressing sound-evoked responses preceded by somatosensory stimuli (Shore, 2005; Kanold et al., 2011; Koehler et al., 2011). Bimodal stimulation can also suppress or enhance subsequent neural activity for up to $1.5 \mathrm{~h}$ in normal animals, an effect termed "bimodal plasticity" (Dehmel et al., 2012b; Koehler and Shore, 2013). Bimodal plasticity expressed by DCN neurons is stimulus timing-dependent with the direction and strength of induced plasticity dependent on the order and relative timing of bimodal stimulation: Hebbian-like unit responses were enhanced when somatosensory preceded auditory stimulation and suppressed when somatosensory followed auditory stimulation. Anti-Hebbianlike unit responses were suppressed when somatosensory preceded auditory stimulation and enhanced when somatosensory followed auditory stimulation (Koehler and Shore, 2013).

Significant changes in bimodal integration and plasticity and their underlying neural circuitry occur after cochlear damage, a leading cause of tinnitus (Lindblad et al., 2011). Cochlear damage decreases auditory nerve terminal counts in the DCN leading to a compensatory increase in excitatory somatosensory terminal counts (Zeng et al., 2012). Furthermore, DCN neurons become more sensitive to somatosensory stimulation after broadband noise overexposure (Shore et al., 2008). In noise-exposed guinea pigs with evidence for tinnitus, sound-evoked responses in neurons with elevated spontaneous firing rates are primarily enhanced after bimodal stimulation (Dehmel et al., 2012b). 
The purpose of the current study was to elucidate the contribution of spike timing-dependent plasticity to tinnitus by evaluating the stimulus timing dependence of bimodal plasticity in a tinnitus model. Here, we exposed guinea pigs to an intense, narrowband noise to induce temporary threshold shifts (TTS) and evaluated gap detection for evidence of tinnitus (Dehmel et al., 2012a; Turner et al., 2012). DCN neural activity was recorded before and 3 and 15 min after bimodal stimulation in a stimulus timing-dependent plasticity protocol. To identify tinnitus correlates, responses were compared between Sham and exposed animals with and without tinnitus. Stimulus timingdependent timing rules shifted from Hebbian-like in Sham animals to antiHebbian-like in noise-damaged animals. Furthermore, enhancement was broader in timing rules from exposed guinea pigs exhibiting tinnitus, whereas suppression was broader in timing rules from exposed animals without tinnitus.

\section{Materials and Methods}

Animals. Female pigmented guinea pigs $(n=16)$ from the Elm Hill colony $(300-400 \mathrm{~g})$ were used in this study. All procedures were performed in accordance with the National Institutes of Health Guidelines for the Use and Care of Laboratory Animals (publication 80-23) and were approved by the University Committee on Use and Care of Animals at the University of Michigan.

Experimental design. This study was designed to assess the effect of noise exposure-induced tinnitus on stimulus timing-dependent bimodal plasticity of sound-evoked responses and spontaneous activity. Guinea pigs were behaviorally tested semiweekly before and after a $2 \mathrm{~h}$ noise exposure (Fig. 1A; $97 \mathrm{~dB}$ noise with $1 / 4$ octave band centered at $7 \mathrm{kHz}$ ) using an acoustic startle-based gap detection assay for tinnitus (Fig. 1B) (Turner et al., 2006; Dehmel et al., 2012a). Guinea pigs were anesthetized with ketamine $(40 \mathrm{mg} / \mathrm{kg})$ and xylazine $(10 \mathrm{mg} / \mathrm{kg})$ during the exposure. Ten guinea pigs were first exposed to the narrowband noise 3-6 weeks after baseline gap detection testing. Six to 8 weeks later, each guinea pig was exposed a second time to the same narrowband noise, as previous results indicated that tinnitus was more likely to develop after repeated noise exposures (Dehmel et al., 2012a, b). The remaining 6 guinea pigs were Sham-exposed at the same time. Auditory brainstem response (ABR) thresholds were measured before beginning gap detection (B), immediately after the first and second noise exposures to assess threshold shift (E1 and E2), one week after each noise exposure to assess recovery of thresholds (R1 and R2), and immediately before unit recordings (F; Fig. $1 C)$. Four to 6 weeks after the second noise exposure, single-unit and multiunit spontaneous activity, rate level functions, and bimodal stimulus timing-dependent plasticity were assessed in an acute DCN recording preparation and compared between Sham and exposed groups and between tinnitus and no tinnitus groups (TGs) (Fig. 1D).

Gap detection testing for tinnitus. The startle-based gap detection assay for tinnitus follows the previously described protocol (Dehmel et al., 2012a). In brief, guinea pigs were placed on top of a piezoelectric force measurement plate to measure movement elicited by a loud broadband noise (the startle stimulus; $115 \mathrm{~dB}, 200-20 \mathrm{kHz}$ ). Each trial consisted of a background noise with (Gap trials) or without (No-Gap trials) a $50 \mathrm{~ms}$ silent gap or pulse embedded $50 \mathrm{~ms}$ before the startle stimulus onset. The $60 \mathrm{~dB}$ background noise was either broadband noise or bandpass-filtered noise with a $2 \mathrm{kHz}$ band and lower cutoff frequencies of $4,8,12,16$, or 20 $\mathrm{kHz}$. Intervals between trials were randomly varied between 18 and $24 \mathrm{~s}$.
Observed startle amplitudes decreased only slightly (by $\sim 10 \%$ ) over multiple testing sessions in both Sham and noise-exposed guinea pigs. For each day of testing, an observation of the normalized startle response was computed as the ratio $\left(\mathrm{A}_{\mathrm{G}} / \mathrm{A}_{\mathrm{NG}}\right)$ where $\mathrm{A}_{\mathrm{G}}$ is the mean amplitude of the startle responses from 10 trials with gap on one day and $\mathrm{A}_{\mathrm{NG}}$ is the mean amplitude of the startle response from 10 trials with no gap on the same day (Fig. 2A). To assess the normalized startle responses within each frequency band for evidence of tinnitus for each frequency band (12 $\mathrm{kHz}$ is shown), the distribution of normalized startle trials from all observations from all animals was analyzed using Gaussian mixture modeling (Statistics Toolbox, MATLAB release 2012b; MathWorks). It was assumed that the normalized startle observations were drawn from one of two distributions, the normal distribution (Fig. $2 B$, black) and the tinnitus distribution, with elevated normalized startle amplitudes (Fig. $2 B$, red). The normalized startle observations were placed into the TG when the probability that the observation belonged to the elevated distribution was $>0.55$ (Fig. $2 C$, red line). Using the threshold established by the Gaussian mixture model, the distributions of normalized startle observations from Sham animals after noise exposure (Sham, Fig. 2D), all animals before noise exposure (Baseline, Fig. 2E), and exposed animals after noise exposure (Exposed, Fig. $2 F$ ) were partitioned into tinnitus and no tinnitus observations. Animals from the exposed group that demonstrated more tinnitus observations than were found during baseline testing were considered to have tinnitus within the tested frequency band. For statistical evaluation, animals with no exposure were assigned to the Sham group, noise-exposed animals with no evidence for tinnitus were assigned to the Exposed-No Tinnitus (ENT) group, and noiseexposed animals with evidence for tinnitus were assigned to the ExposedTinnitus (ET) group. Prepulse inhibition was assessed in the same manner as gap detection. All groups of animals showed no differences in prepulse inhibition before and after the noise damage. This result was taken to mean that baseline temporal processing was unchanged by the noise damage; therefore, any changes in gap detection were as a result of the tinnitus "filling the gap" and not because of a temporal processing dysfunction or hearing loss.

Surgical approach for neural recordings. Guinea pigs were anesthetized (subcutaneous injection of $100 \mathrm{mg} / \mathrm{kg}$ ketamine and $10 \mathrm{mg} / \mathrm{kg}$ xylazine; at the incision site a subcutaneous injection of lidocaine, $14 \mathrm{mg} / \mathrm{kg}$ ) and ophthalmic ointment applied to their eyes. Their heads were fixed in a Kopf stereotaxic frame using a bite bar, and hollow ear bars were placed into the ear canals. Core temperature was maintained at $38^{\circ} \mathrm{C}$. A left craniotomy was performed, and a small amount of cerebellum was aspirated (leaving paraflocculus intact) to allow for visual placement of the 
A

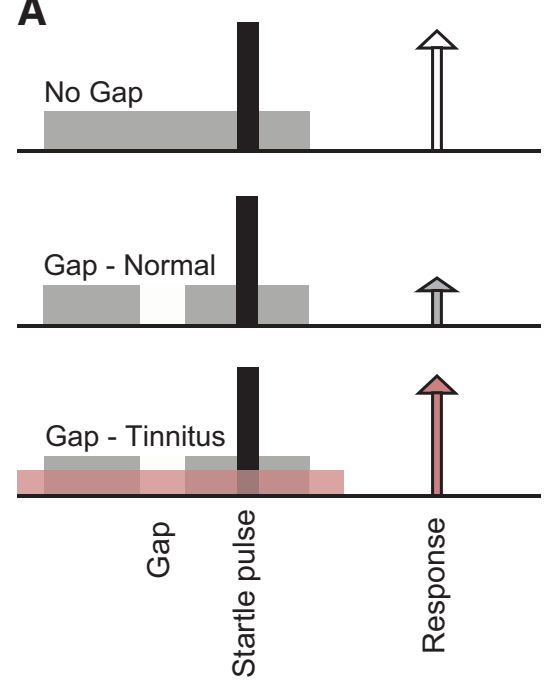

B
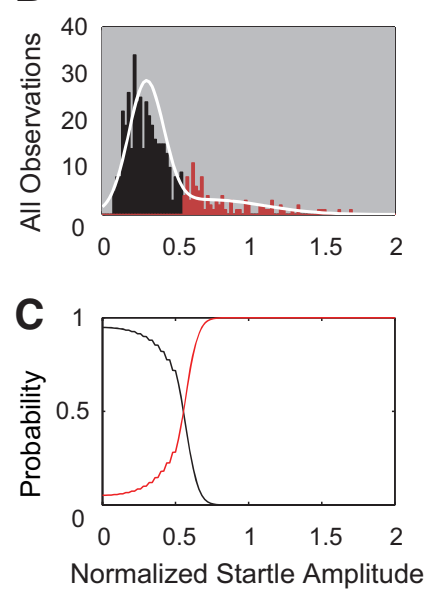

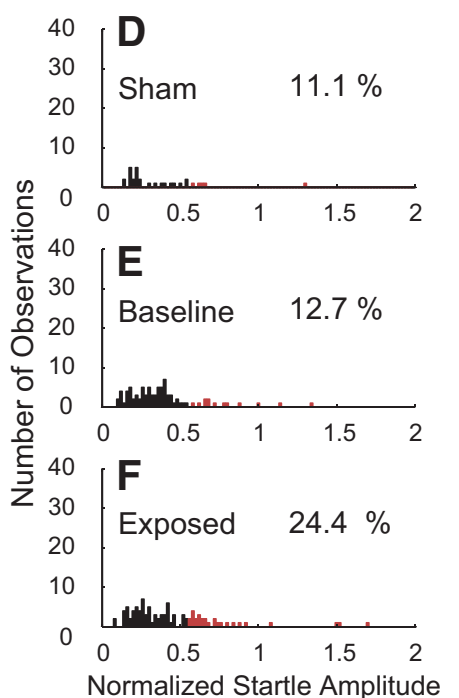

Figure 2. A, Schematic describing the startle-based gap-detection assay for tinnitus. No gap (top row) and gap trials ( $50 \mathrm{~ms}$ gap, $50 \mathrm{~ms}$ before the startle sound; bottom two rows) are presented to the animal. Each trial consists of a continuous $60 \mathrm{~dB}$ background sound (gray bar) with a $10 \mathrm{~ms}, 115 \mathrm{~dB}$ startle pulse embedded (black bar). The guinea pig startles in response to the startle stimulus, with the amplitude of the response shown by the height of each arrow. In animals without tinnitus, the gap introduces a suppression of the startle response (middle row). In animals with tinnitus, the gap is filled by the tinnitus (pink), and the startle response shows less reduction relative to the no gap startle response (white arrow). $\boldsymbol{B}-\boldsymbol{F}$, Gaussian mixture model analysis partitioning the normalized startle distribution into normal and tinnitus distributions. $\boldsymbol{B}$, Histogram of the normalized startle distribution (white line) partitioned into two distributions: no evidence for tinnitus (black bars) and evidence for tinnitus (red bars). C, The posterior probabilities that normalized startle values belong to the tinnitus or nontinnitus distributions. $\boldsymbol{D}$, Histogram of the partitioned distribution of postexposure normalized startle observations for Sham animals. $\boldsymbol{E}$, Histogram of the partitioned distribution of normalized startle observations for baseline (preexposure) observations from Sham and exposed animals. $\boldsymbol{F}$, Histogram of the partitioned distribution of postexposure normalized startle observations from exposed animals. $\boldsymbol{D}-\boldsymbol{F}$, Percentages of observations placed into the TG is shown on each panel.

recording electrode. Supplemental doses of ketamine and xylazine (i.m.) were administered at least hourly when indicated by response to a toe pinch. The guinea pig's physiological condition was monitored by assessment of body temperature, respiration rates, heart rates, and unit thresholds. After the completion of neural recording, the guinea pig was killed by intraperitoneal injection of sodium pentobarbitol followed by decapitation.

Electrode placement. A concentric bipolar stimulating electrode (FHC) was placed stereotaxically into spinal trigeminal nucleus (Sp5) after being dipped in Fluorogold (10 degrees below horizontal, $0.28 \pm 0.03 \mathrm{~cm}$ lateral from midline, $0.25 \pm 0.02 \mathrm{~cm}$ caudal from transverse sinus, $0.9 \pm$ $0.1 \mathrm{~cm}$ below the surface of cerebellum). Postmortem reconstruction confirmed electrode locations in Sp5. A four shank, 32 channel siliconsubstrate electrode (site spacing $=100 \mu \mathrm{m}$, shank pitch $=250 \mu \mathrm{m}$, site area $=177 \mu \mathrm{m}^{2}$, impedance $=1-3 \mathrm{mOhms}$, NeuroNexus) was placed with the tips $0.8-1.0 \mathrm{~mm}$ below the surface of the DCN with shanks rostral-to-caudal approximately within an iso-frequency layer. If the top sites on each shank did not demonstrate a response to sound, the electrode was lowered until they responded to noise.

Auditory and somatosensory stimulation. Cosine window-gated tone signals (50 ms duration, $2 \mathrm{~ms}$ rise/fall time) were generated using Open Ex and an RX8 DSP (TDT) with 12 bit precision and sampling frequency set at $100 \mathrm{kHz}$. A shielded speaker (DT770, Beyer) driven by an HB7 amplifier (TDT) delivered sound through a hollow earbar to the left year. The system response was measured with a condenser microphone attached to the hollow earbar by a $1 / 4$ " long tube approximating the ear canal. Sound levels were adjusted to account for the system response using a programmable attenuator (PA5, TDT) to deliver calibrated levels ( $\mathrm{dB}$ SPL) at frequencies from $200 \mathrm{~Hz}$ to 24 $\mathrm{kHz}$. Neurons in somatosensory brainstem nuclei (Sp5) known to project to DCN were activated by three biphasic (100 $\mu \mathrm{s} /$ phase) current pulses at $1000 \mathrm{~Hz}$ delivered to Sp5 through a concentric bipolar electrode (Shore et al., 2008). Responses to unimodal Sp5 stimulation were assessed before any bimodal stimulation and classified as excitatory, inhibitory, or having a complex excitatory/inhibitory pattern. The current amplitude was set to the highest level (range 50-70 $\mu \mathrm{A}$ ) that did not elicit movement artifact.
Assessment of stimulus timing-dependent bimodal plasticity. Stimulus timing-dependent plasticity was assessed in all guinea pigs using an in vivo bimodal plasticity induction protocol (Dehmel et al., 2012a; Koehler and Shore, 2013). In short, spontaneous activity and responses to unimodal tone stimuli were recorded at three time points: before and 3 and $15 \mathrm{~min}$ after the bimodal stimulation protocol. Three and $15 \mathrm{~min}$ time points were selected to confirm the maximal bimodal effect that was previously observed at $15 \mathrm{~min}$ with recovery occurring after $90 \mathrm{~min}$ (Koehler and Shore, 2013). Responses from the $15 \mathrm{~min}$ time point were used for all bimodal plasticity measurements. Responses to tones were assessed by computing the mean firing rate over the $50 \mathrm{~ms}$ window corresponding to the duration of the tone. Spontaneous activity was assessed by computing the mean firing rate over $2-3$ min of spiking activity in the absence of sound. The bimodal stimulation protocol consisted of 300 trials of the $50 \mathrm{~ms}$ tones combined with Sp5 activation presented at $2 \mathrm{~Hz}$. The bimodal interval (BI) was defined as the difference between the tone stimulus and the Sp5 stimulus onset time. Thus, negative BIs indicate sound-leading Sp5 stimulation, and positive BIs indicate Sp5-leading sound stimulation. Stimulus timing dependence was assessed by varying the BI and measuring the change in unimodal toneevoked firing rates before and after bimodal stimulation. The recording block was repeated with the $\mathrm{BI}$ randomly selected from the following list: $-40,-20,-10,10,20$, or $40 \mathrm{~ms}$. In control recording blocks, unimodal sound-evoked responses were assessed following an identical protocol, except with unimodal tone or Sp5 stimuli replacing the bimodal stimuli to test unimodal plasticity.

To assess recovery after bimodal stimulation, responses to unimodal tones were measured every 15-30 min after the final bimodal stimulation block for up to $2 \mathrm{~h}$. Timing rules for principal cell units (excluding units with type II receptive fields) were classified as Hebbian, anti-Hebbian, suppressing, or enhancing by comparing the mean change in firing rate (i.e., the firing rate before bimodal stimulation subtracted from the firing rate after bimodal pairing) when the Sp5 stimulus preceded the sound and when the Sp5 stimulus followed the sound. Timing rule classification corresponded to that used previously (Koehler and Shore, 2013). For comparison between Sham, $\mathrm{ET}$, and ENT animals, spontaneous firing rates were measured from the first recording block before any bimodal stimulation. 
Spike detection and sorting. Voltages from each site were digitized by a $\mathrm{PZ} 2$ preamp (Fs $=$ $12 \mathrm{kHz}, \mathrm{TDT})$ and bandpass filtered $(300 \mathrm{~Hz}$ to $3 \mathrm{kHz}$ ). Online spike detection used a voltage threshold set 2.5 SDs above background noise (RZ2, TDT). Timestamps and waveform snippets were saved to a PC and sorted using principal components of the waveform shape and EM-Dist cluster analysis with fixed variance (95\%) and 5 clusters (Plexon Offline Sorter). Cluster distinctness was confirmed with pairwise cluster statistics ( $p>0.05$; Plexon Offline Sorter) and visually by a trained observer. When a spike was present in a $1 \mathrm{~ms}$ window across $80 \%$ of channels, any spikes within that window were considered artifact and removed from further analysis. The waveform shapes, amplitudes, and response properties of multiunit clusters in this study were consistent over the duration of the recording.

Statistical analyses. Significant bimodal plasticity was identified using a paired measurement Student's $t$ test for the number of spikes measured on each trial before and after bimodal stimulation. Timing rules were constructed and classified for each individual unit as Hebbian, anti-Hebbian, suppressing, or enhancing. The proportion of timing rule types were compared between units from Sham and exposed animals or between units from Sham, ET, and ENT units and tested for significance using a $2 \times 2$ or $2 \times 3 \chi^{2}$ test, respectively, for independence. Differences between mean population timing rules were tested using 2-way ANOVAs followed by Tukey-Kramer's post hoc tests. All statistical tests were run in MATLAB using the Statistics toolbox. $p$ values for post hoc tests were computed using a custom MATLAB routine that identifies the lowest groupwise $p$ value for which there is a significant difference after applying Tukey-Kramer.

\section{Results}

\section{Narrowband noise exposure centered at $7 \mathrm{kHz}$ induced temporary threshold shifts between 7 and $16 \mathrm{kHz}$}

Noise exposure induced a TTS as demonstrated by ABR thresholds. Thresholds in the exposed ear (Fig. $3 A$ ) but not the unexposed ear (Fig. $3 B$ ) were elevated immediately after exposure and recovered to baseline by 1 week after noise exposure. Maximum threshold elevation was (mean $\pm \mathrm{SD}$ ) $35 \pm 3.5 \mathrm{~dB}$ at $9 \mathrm{kHz}$ after the first exposure and $19 \pm 2.1 \mathrm{~dB}$ at $10 \mathrm{kHz}$ after the second exposure with thresholds elevated in a band from the exposure frequency to 2 octaves above the exposure frequency. ABR thresholds in Sham-exposed guinea pigs were not elevated above baseline in either ear (Fig. 3C,D).

\section{Narrowband noise exposure induced tinnitus in the 12-14 $\mathrm{kHz}$ band in $60 \%$ of guinea pigs}

Gap-induced prepulse inhibition of acoustic startle was used to assess each guinea pig for evidence of a frequency-specific tinnitus percept. During baseline startle testing, all guinea pigs exhibited normal gap detection with smaller startle responses when there was a gap than when there was no gap. All guinea pigs exhibited normalized startle responses $<0.5$ during baseline, with the normalized startle response defined as the ratio of the startle response amplitude with gap prepulse $\left(\mathrm{A}_{\mathrm{G}}\right)$ to the startle response amplitude without gap $\left(\mathrm{A}_{\mathrm{NG}}\right)$. Impaired gap detection, which was considered evidence for tinnitus, was identified by significantly elevated normalized startle responses. After the TTS-inducing noise exposure, $60 \%$ of exposed guinea pigs were identified as having tinnitus in the $12-14 \mathrm{kHz}$ band, half of which also showed evidence for tinnitus in the $4-8,8-10$, or $16-18 \mathrm{kHz}$ bands (Fig. $4 A$; see Materials and Methods). Guinea pigs with evidence for tinnitus in the $12-14 \mathrm{kHz}$ bands were thus placed into the ET group. The remaining $40 \%$ of exposed guinea pigs that showed no evidence for tinnitus in any tested frequency band were placed into the ENT group, whereas the Sham animals were considered as a separate group (Sham).

To validate the ET and ENT groupings, gap detection ability was compared between all exposed and Sham guinea pigs (Fig. $4 B$ ), and between the ET, ENT, and Sham guinea pigs (Fig. 4C). The normalized startle response was not significantly elevated for any frequency band in all exposed guinea pigs (Fig. 4B). However, normalized startle responses were significantly elevated, indicating impaired gap detection ability, for the 4-6, 8-10, and 12-14 $\mathrm{kHz}$ bands in the ET group but not in the ENT group (Fig. $4 C$ ). The normalized startle response was not significantly elevated for the BBN background signal or the $16-18 \mathrm{kHz}$ background signal either in the ET group or the ENT group (Fig. 4C).

\section{Bimodal plasticity timing rules were predominantly anti- Hebbian and suppressing in noise-exposed animals}

Previously, we measured the stimulus timing dependence of bimodal plasticity and demonstrated predominantly Hebbian-like timing rules in normal animals (Koehler and Shore, 2013). To separate noise exposure-driven changes in neural mechanisms from those associated with tinnitus, stimulus timing-dependent bimodal plasticity was first compared between principal cell units from Sham ( $n=100$ units) and Exposed ( $n=288$ units) guinea pigs and then between exposed animals that developed tinnitus (ET) or did not develop tinnitus (ENT). Bimodal plasticity was assessed by identifying significant changes in sound-evoked average firing rates 15 min after bimodal stimulation (Fig. 5A). The dependence of bimodal plasticity on stimulus timing was confirmed by repeatedly measuring bimodal plasticity using the protocol in Figure $5 \mathrm{~A}$ and varying the $\mathrm{BI}(10,20$, and $40 \mathrm{~ms})$ and order (Sp5 or tone leading) in the bimodal pairing protocol. BIs were classified as eliciting significant bimodal plasticity if the firing rates before and $15 \mathrm{~min}$ after were significantly different ( $t$ test, $p<0.05$ ).

Timing rules were constructed for each unit by plotting the change in sound-evoked firing rates observed 15 min after vari- 
A

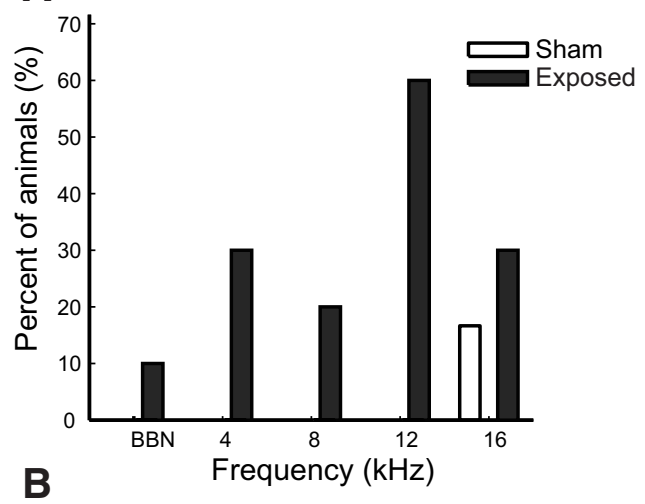

B
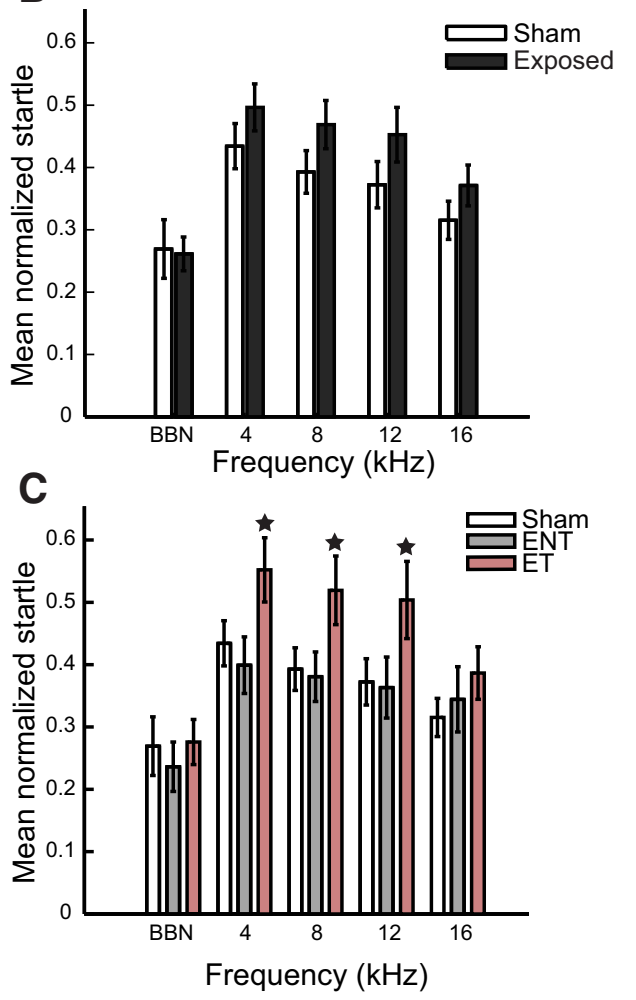

Figure 4. Elevated normalized startle responses demonstrate tinnitus in the 4 to $16 \mathrm{kHz}$ bands. $A$, Percentage of Sham (white bars) and exposed (black bars) guinea pigs that show evidence for tinnitus in different frequency bands. $\boldsymbol{B}$, Normalized startle response amplitudes in each frequency band for exposed animals (black bars) compared with Sham animals (white bars). $C$, Normalized startle response amplitudes in each frequency band for tinnitus animals (ET, red bars) compared with animals without tinnitus (ENT, gray bars) and Sham animals (white bars). B, C, Error bars indicate $95 \%$ confidence intervals. $\star$ Significantly different from other bars within the same frequency band.

ous bimodal pairing orders and intervals. These timing rules were classified as Hebbian-like ( $n=132$; examples from Sham and noise-exposed animals shown in Fig. $5 B$ ), anti-Hebbian-like ( $n=$ 69; examples from Sham and noise-exposed animals shown in Fig. $5 C)$, enhancing $(n=44)$, or suppressing $(n=143)$. In units with Hebbian-like timing rules, sound-evoked firing rates increased after bimodal stimulation when Sp5 stimulation preceded tone onset and decreased after bimodal stimulation when Sp5 stimulation followed tone onset (Fig. 5B). The temporal window for enhancing bimodal plasticity is broader in this unit from a noise-exposed animal from the ET group, with enhancement observed after BIs of $-10,10$, and $20 \mathrm{~ms}$. In contrast, in the unit from the Sham animal, enhancement is only observed with BIs of
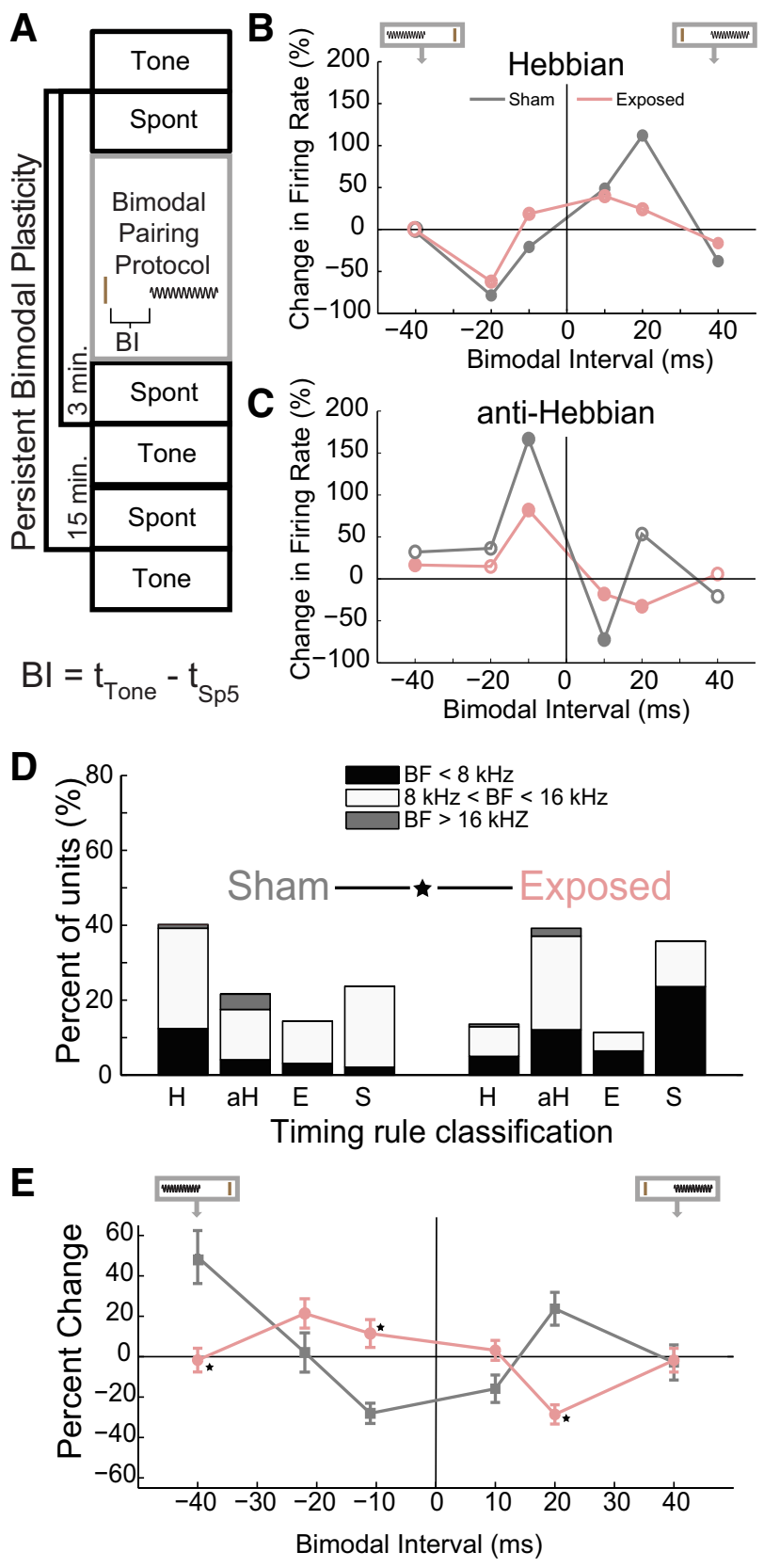

Figure 5. Bimodal plasticity timing rules are significantly different between Sham and noiseexposed guinea pigs. $A$, Diagram representing the bimodal plasticity measurement protocol. Spontaneous activity (Spont) and responses to tones (Tone) were recorded before and 3 and 15 min after the bimodal pairing protocol. The bimodal pairing protocol consisted of repeated presentations of an Sp5 stimulus (brown line) and a short tone burst (black sinusoid) with varied Bls. Bl is defined below the diagram, with $\mathrm{t}_{\text {Tone }}$ representing the onset time of the tone stimulus and $\mathrm{t}_{\mathrm{sp}}$ representing the onset time of the $\mathrm{Sp} 5$ stimulus. $\boldsymbol{B}$, Two examples of single-unit Hebbian timing rules: one from a Sham (gray) and one from a noise-exposed (pink) guinea pig. A diagram at the top of the panel demonstrates the relative order of $S p 5$ and sound stimuli (also in $E$ ). The brown vertical line represents the $S p 5$ stimulus and the sinusoid represents the tone stimulus. C, Two examples of single-unit anti-Hebbian timing rules: one from a Sham and one from a noise-exposed guinea pig. $B, C$, Filled circles represent significant changes in sound-evoked firing rates. $\boldsymbol{D}$, The percentage of principal units that showed Hebbianlike (H), anti-Hebbian-like (aH), enhancing (E), and suppressing $(\mathrm{S})$ timing rules from Sham (left) and noise-exposed (right) animals. Stacked bars indicate units from below (black), within (white), and above (gray) the damaged frequency region. Data were obtained at the 15 min time point. $\star$ Significant differences in the distribution of timing rules between Sham and exposed animals. $\boldsymbol{E}$, Timing rules shifted from Hebbian in Sham animals to anti-Hebbian in exposed animals. Mean timing rules showing bimodal plasticity of sound-evoked firing rates for units from Sham (gray) and exposed (pink) guinea pigs. Mean timing rules were computed for all measurements from principal cell units. Error bars indicate SEM. $\star$ Significant differences ( $p<0.05$; Tukey-Kramer's post hoc test). 
10 and 20 ms. In units with anti-Hebbianlike timing rules, sound-evoked firing rates increased 15 min after Sp5 stimulation followed tone onset and decreased 15 min after Sp5 stimulation preceded tone onset (Fig. 5C). Responses in the remaining units were only enhanced or suppressed after bimodal stimulation at the tested BIs (individual units not shown).

Units from Sham animals were distributed among the timing rule classes in proportions similar to normal animals (Koehler and Shore, 2013), with most units showing Hebbian-like timing rules (Fig. 5 D, left column). In contrast, after noise exposure, units with anti-Hebbian and suppressive timing rules were significantly more prevalent $\left(\chi^{2}\right.$ proportion test; Sham vs Exposed; df $=3 ; \chi^{2}=25.2564$; $p<0.0001)$ than Hebbian or enhancing units (Fig. 5D, right column). Further breakdown of units into those with BFs within the TTS frequency region $(8-16$ $\mathrm{kHz})$ and those outside these regions $(<8$ $\mathrm{kHz}$ and $>16 \mathrm{kHz}$ ) reveal that units within the TTS frequency region comprised the highest percentages of units in the anti-Hebbian class.

Mean timing rules estimate the effect of somatosensory-auditory pairing at specific intervals on DCN population activity. Mean population timing rules from all Sham and exposed units 15 min after bimodal stimulation revealed a shift in the population timing rules from Hebbianlike to anti-Hebbian-like in the noiseexposed animals (Fig. 5E). In Sham animals, the mean population timing rule was Hebbian-like, with enhancement of sound-evoked firing rates when Sp5 preceded sound stimulation (positive values) and suppression when $\mathrm{Sp} 5$ stimulation followed sound stimulation (Fig. 5E, gray, negative values). In noiseexposed animals, the reverse occurred, with suppression of sound-evoked firing rates for $\mathrm{Sp} 5$ preceding sound stimulation and enhancement with Sp5 after sound stimulation (Fig. 5E, pink). A two-way ANOVA with Exposure Group (Group) and BI revealed a significant main effect of $\mathrm{BI}$ and a significant interaction between BI and Exposure Group (Group $\mathrm{F}(1)=1.38, p=$ 0.240 ; $\mathrm{BI} \mathrm{F}(5)=5.37, p<0.001$; Exposure Group $\times \mathrm{BI} \mathrm{F}(5)=$ $14.47, p<0.001)$. BIs for which there were significant differences between Exposure Groups according to Tukey-Kramer's post hoc tests are designated in Figure $5 E$ by stars.

Anti-Hebbian bimodal enhancement was broader in guinea pigs exhibiting tinnitus, whereas suppressive bimodal plasticity was broader in animals without tinnitus

To establish tinnitus-specific differences in bimodal stimulus timing-dependent plasticity, we compared responses between Sham ( $n=100$ units), ENT ( $n=63$ units), and ET ( $n=225$ units) guinea pigs before and $15 \mathrm{~min}$ after bimodal stimulation of varying orders and intervals. Mean population timing rules for ET, ENT, and Sham units revealed that bimodal plasticity was converted from Hebbian-like to anti-Hebbian-like timing rules in both the ET and ENT groups (Fig. 6A). In the ET animals, there were more BIs at which enhancement occurred $(-40,-20,-10$, and $10 \mathrm{~ms}$ ) than in the ENT group (only $-20 \mathrm{~ms}$ ), revealing a broadening of the timing rules for the enhancement phase of the curve in the ET animals. Although firing rate suppression was observed at $20 \mathrm{~ms}$ for both ET and ENT animals, there was a broadening of the suppressive phase of the curve in the ENT animals, with suppression at both 10 and $20 \mathrm{~ms}$ compared with only $20 \mathrm{~ms}$ in the ET animals (Fig. 6A). The broadening of the timing rules in the enhancement phase in ET animals and in the suppressive phase in ENT animals was in contrast to narrow, Hebbian-like timing rules in Sham animals, in which maximal enhancement and suppression were found at BIs of $20 \mathrm{~ms}$ and $-10 \mathrm{~ms}$, respectively. A two-way ANOVA with TG and BI revealed significant main effects of TG and BI and a significant interaction between BI and Exposure Group ( $\mathrm{TG} \mathrm{F}(2)=4.02$, $p=0.018$; BI F $(5)=4.72, p<0.001 ; \mathrm{TG} \times \mathrm{BIF}(10)=7.34, p<$ 0.001). BIs for which there were significant differences between TGs according to Tukey-Kramer's post hoc tests are shown as stars in Figure $6 \mathrm{~A}$.

Corresponding with the shifts in population timing rules, anti-Hebbian-like units were most common in ET animals, whereas suppressive units were predominant in ENT animals (Fig. $6 B ; \chi^{2}=52.82 ; \mathrm{df}=11 ; p<0.001$; Fig. $6 B$, stars). The shift 
A

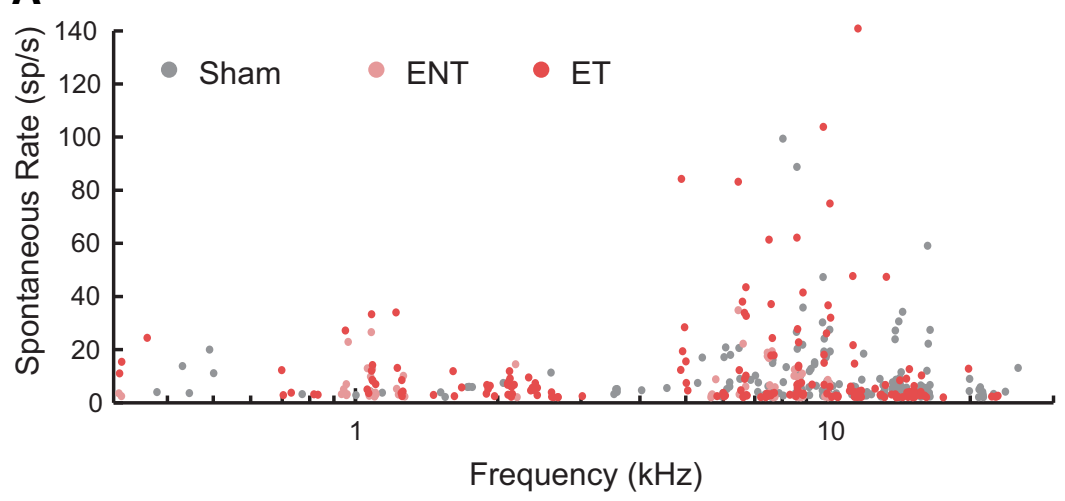

B

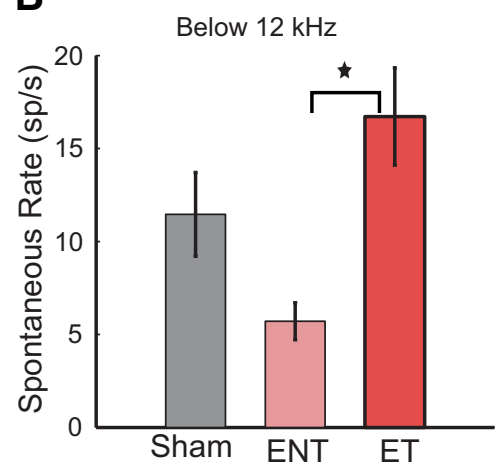

C

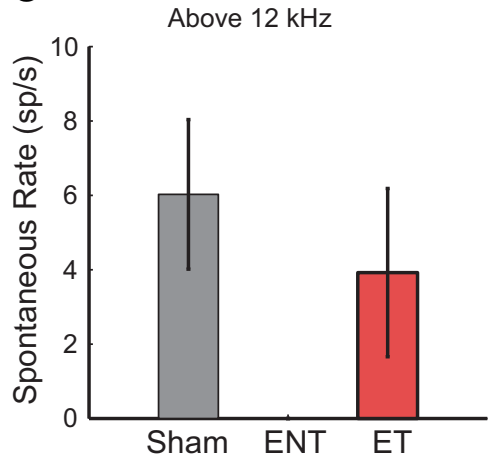

D

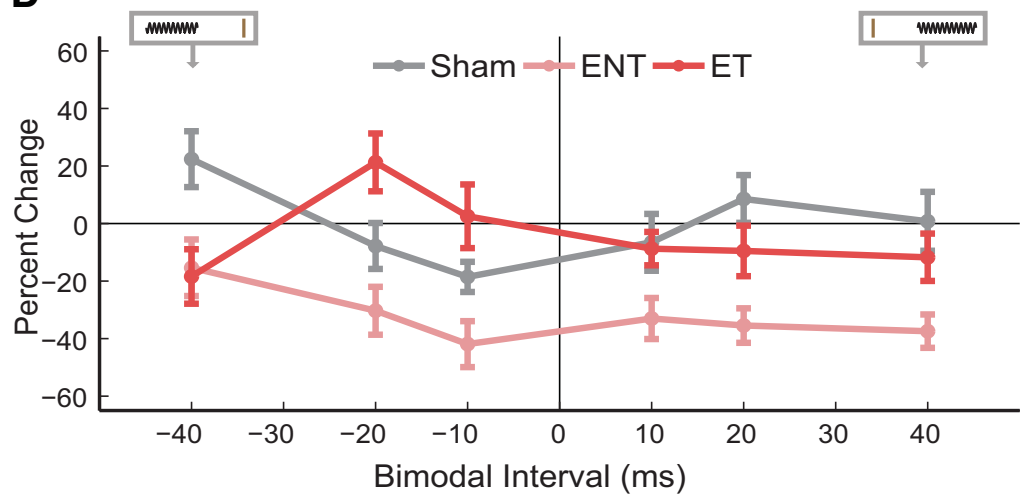

Figure 7. Spontaneous firing rates show hyperactivity in ET but not ENT guinea pigs. $A$, Spontaneous firing rates before any bimodal stimulation for each unit as a function of each unit's best frequency for Sham (gray), ENT (pink), and ET (red) units. B, Mean spontaneous rates for units with best frequencies $<12 \mathrm{kHz}$. C, Mean spontaneous rates for units with best frequencies $>12 \mathrm{kHz}$. SR, Spontaneous rates. $\star$ Significant differences $(p=0.003)$. $D$, Mean timing rules show bimodal plasticity of spontaneous firing rates for units from Sham (gray), ENT (pink), and ET (red) guinea pigs. Mean timing rules were computed for all measurements from presumed principal cell units. Error bars indicate SEM.

toward anti-Hebbian-like units in ET animals was more prominent in units within the tinnitus frequency bands.

Anti-Hebbian bimodal plasticity of spontaneous rates was dominant in noise-exposed guinea pigs exhibiting tinnitus, whereas suppressive bimodal plasticity of spontaneous rates was dominant in animals without tinnitus

Tinnitus is associated with spontaneous hyperactivity in the DCN and other auditory structures (Komiya and Eggermont, 2000; Brozoski et al., 2002; Kaltenbach et al., 2004; Dehmel et al., 2012b). Spontaneous firing rates measured before any bimodal stimulation revealed elevated spontaneous firing rates in the ET group in frequency regions with threshold shifts and evidence for tinnitus $(<12 \mathrm{kHz}$; Fig. $7 A, B)$ but not $>12 \mathrm{kHz}$ (Fig. $7 A, C$ ). It is therefore important to assess the influence of bimodal stimulation on subsequent spontaneous activity in Sham, ENT, and ET animals. Figure $7 D$ plots the change in spontaneous activity observed in DCN neurons 15 min after various bimodal pairing orders and intervals in the three groups. These timing rules constructed from changes in spontaneous rates in units from ENT animals were generally suppressive. In contrast, units from ET animals exhibited anti-Hebbian-like timing rules with enhancement at the $-20 \mathrm{~ms}$ intervals and less suppression at all positive intervals than the ENT animals. A two-way ANOVA with TG and BI revealed significant main effects of TG and a significant interaction between $\mathrm{BI}$ and Exposure Group $(\mathrm{TG} F(2)=14.06, p<0.0001 ; \mathrm{BI}$ $\mathrm{F}(5)=1.12, p=0.35 ; \mathrm{TG} \times \mathrm{BI} F(10)=$ 2.41, $p=0.008)$.

\section{Responses to Sp5 stimulation were predominantly inhibitory in normal animals but excitatory in animals with tinnitus}

Given that noise exposure can affect the auditory (Kujawa and Liberman, 2009) and somatosensory (Shore et al., 2008; Zeng et al., 2012) drive to the DCN, we evaluated tinnitus-associated changes in responses to unimodal somatosensory stimulation. Responses to Sp5 stimulation alone were recorded to compare the distributions of excitatory, inhibitory, and complex unimodal Sp5 responses between Sham, noise-exposed, and TGs (Fig. 8). Unimodal Sp5 responses were more likely to be excitatory (green) and less likely to be inhibitory (red) in ET animals than in Sham and ENT animals. In contrast, unimodal Sp5 responses were more likely to be complex (E/In; blue) in ENT animals. These responses to somatosensory unimodal stimulation suggest that somatosensory excitatory drive to the DCN is strengthened in animals with tinnitus.

\section{Bimodal stimulation was more effective at inducing plasticity than unimodal stimulation}

The contribution of bimodal stimulation to plasticity of soundevoked responses was compared with the effects of repeated unimodal sound (Fig. 9A,B) or unimodal $S p 5$ (Fig. 9C,D) stimulation on subsequent sound-evoked responses. Bimodal stimulation induced significantly more enhancement and suppression than unimodal tone stimulation for all groups, although the effect was more pronounced in units from ENT and ET animals than in Sham animals (Fig. 9A, B; two-way ANOVA for enhancement; TG $\mathrm{F}(2)=4.90, p=0.008$; Stimulus $\mathrm{F}(1)=14.84, p<$ 0.001 ; TG $\times$ Stimulus $F(2)=0.47, p=0.63$; Figure $9 A$ stars 


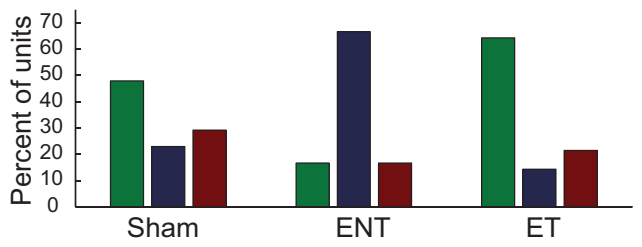

Figure 8. Responses to Sp5 stimulation shift from predominantly excitatory to predominantly suppressive in tinnitus animals. The percentage of units showing excitatory (green), complex (blue), and inhibitory (red) responses to unimodal Sp5 stimulation.
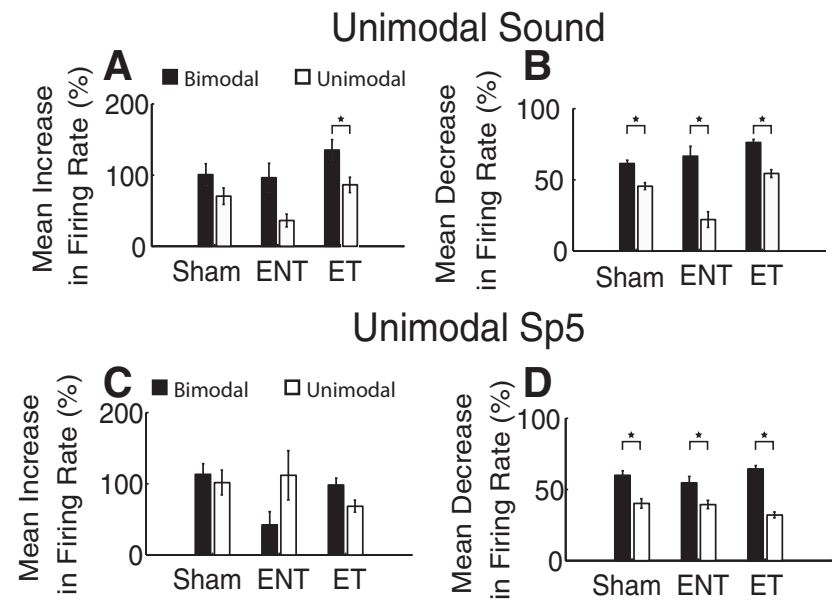

Figure 9. Bimodal stimulation influences long-term firing rates more than unimodal stimulation, especially in animals with tinnitus. $A$, Mean increases in firing rate after bimodal (black) and unimodal sound (white) stimulation from Sham, ENT, and ET animals. $\boldsymbol{B}$, Mean decreases in firing rate after bimodal (black) and unimodal sound (white) stimulation from Sham, ENT, and ET animals. C, Mean increases in firing rate after bimodal (black) and unimodal Sp5 (gray) stimulation from Sham, ENT, and ET animals. D, Mean decreases in firing rate after bimodal (black) and unimodal Sp5 (gray) stimulation from Sham, ENT, and ET animals. Error bars indicate SEM. Stars indicate significance (see text).

indicate Tukey-Kramer's post hoc test for unimodal to bimodal comparisons; two-way ANOVA for suppression; $\mathrm{TG} \mathrm{F}(2)=18.0$, $p<0.001$; Stimulus $\mathrm{F}(1)=92.6, p<0.001$; TG $\times$ Stimulus $\mathrm{F}(2)=6.86, p=0.001$; Figure $9 B$ stars indicate Tukey-Kramer's post hoc test for unimodal to bimodal comparisons).

Similarly, bimodal stimulation induced more enhancement than unimodal Sp5 stimulation (Fig. 9C) in units from ET animals. However, unimodal $S p 5$ stimulation induced greater enhancement for units from ENT animals (Fig. 9C). A two-way ANOVA with TG and stimulus type (Unimodal Sp5 vs Bimodal stimulation) revealed no significant main effects or interactions $(\mathrm{TGF}(2)=2.10, p=0.12$; Stim Type $\mathrm{F}(1)=0.38, p=0.54$; $\mathrm{TG} \times$ Stim Type $\mathrm{F}(2)=2.91, p=0.056)$. Bimodal stimulation induced significantly more suppression than unimodal Sp5 stimulation for all groups (Fig. 9D), although the effect was strongest for units from ET animals in which the bimodal suppression increases (two-way ANOVA; TG F $(2)=0.42, p=0.66$; Stim Type $\mathrm{F}(1)=$ 81.0, $p<0.001$; TG $\times$ Stim Type $\mathrm{F}(2)=5.15, p=0.006$; Figure 9 stars indicate Tukey-Kramer's post hoc test for unimodal to bimodal comparisons).

\section{Discussion}

Bimodal auditory-somatosensory plasticity in the $\mathrm{DCN}$ in vivo is stimulus timing-dependent (Koehler and Shore, 2013), consistent with in vitro studies of spike timing-dependent plasticity (STDP) at parallel-fiber synapses (Tzounopoulos et al., 2004) that convey somatosensory information to the DCN (Zhou and Shore, 2004; Zhou et al., 2007). Alterations in timing rules governed by stimulus timing-dependent plasticity have been associated with pathology, such as Parkinson's disease and cognitive disorders (Aosaki et al., 2010; Meredith and Mansvelder, 2010). Here, we demonstrate changes in stimulus timing-dependent bimodal plasticity in a model of noise-exposure induced tinnitus. We showed that bimodal plasticity timing rules were more likely to be anti-Hebbian in guinea pigs with tinnitus than those without tinnitus or in Shams. Furthermore, the timing window for enhancement was broader in animals that developed tinnitus. In contrast, exposed animals that did not develop tinnitus exhibited primarily suppressive timing rules and broader timing windows for suppression in units with anti-Hebbian like rules.

\section{Noise exposure causes temporary threshold shifts and tinnitus}

Noise exposure is a leading cause of tinnitus in humans, with the tinnitus frequency usually close to the frequency region of greatest hearing loss (Heller, 2003). In the present study, narrowband noise centered at $7 \mathrm{kHz}$ produced unilateral TTS with threshold shifts at $7-16 \mathrm{kHz}$ and tinnitus in the $12-14 \mathrm{kHz}$ bands, consistent with earlier reports of tinnitus above the noise exposure frequency (Turner et al., 2006; Bauer et al., 2008).

\section{Alterations in bimodal stimulus timing-dependent plasticity are associated with noise exposure and tinnitus}

Noise-exposure shifts bimodal timing rules from Hebbian to anti-Hebbian or suppressing. Compared with those that did not develop tinnitus, the exposed animals with tinnitus exhibited several striking characteristics: (1) population plasticity was more likely to be governed by stimulus timing-dependent (primarily anti-Hebbian) rules than suppressive or enhancing plasticity; (2) timing rules were broader in the enhancing phase of the curve but narrower in the suppressive phase; (3) plasticity of spontaneous activity shifted toward enhancement at all BIs; and (4) bimodal plasticity was stronger than unimodal plasticity. These tinnitusassociated changes in bimodal stimulus timing-dependent plasticity suggest that somatosensory inputs have a greater influence on DCN neural activity in noise-exposed animals, with a bias toward enhancement in animals that developed tinnitus and a bias toward suppression in those that did not, consistent with previous findings (Dehmel et al., 2012b).

\section{Bimodal stimulus timing-dependent plasticity is a potential mechanism for tinnitus}

Bimodal STDP in the DCN has been proposed as a mechanism to identify spatiotemporal patterns in auditory nerve activity that are correlated with somatosensory inputs (Roberts and Portfors, 2008). In the normal system, parallel fiber synaptic activity modulates the responsivity of DCN neurons to auditory nerve activity by reducing input resistance and broadening synaptic integration (Tzounopoulos et al., 2007; Doiron et al., 2011). Thus, narrow STDP timing rules that potentiate or depress these synapses can heighten or suppress DCN responsivity to auditory nerve inputs (Masquelier et al., 2008) that are tightly correlated with somatosensory events (Sawtell, 2010). In tinnitus animals, the broader temporal window for enhancing plasticity (Fig. 6A) would increase the likelihood of a somatosensory event triggering longterm potentiation through Hebbian or anti-Hebbian STDP. This would lead to heightened responsivity to spontaneous and driven auditory nerve and parallel fiber spiking. In the present model, it is more likely to arise from the somatosensory system, as somato- 
sensory inputs are more numerous after noise damage (Zeng et al., 2009, 2012). A similar process operates in visual cortex, where broadened STDP timing rules after visual deprivation cause longterm potentiation of spontaneous inputs (Guo et al., 2012).

These alterations in bimodal plasticity, acting cooperatively with increased somatosensory synaptic contacts and decreased granule cell resistance after noise exposure (Zeng et al., 2009, 2012; Pilati et al., 2012), could be responsible for strengthening somatosensory drive, leading to spontaneous hyperactivity in DCN neurons. The corresponding decrease in bimodal suppression of spontaneous activity in tinnitus animals would further enhance hyperactivity. Because the DCN has been identified as necessary for the generation of tinnitus (Brozoski et al., 2012), spontaneous hyperactivity driven by these mechanisms could be a neural representation of tinnitus (Kaltenbach and McCaslin, 1996).

\section{Potential mechanisms underlying noise-induced changes in stimulus timing-dependent plasticity}

The influence of noise exposure on DCN microcircuitry, STDP neuromodulators, and synaptic plasticity mechanisms must be considered in the interpretation of the in vivo changes in stimulus timing-dependent plasticity demonstrated here.

First, a reduction in glycinergic inhibition after noise exposure (Suneja et al., 1998a, b) and tinnitus (Wang et al., 2009) could shift the balance of sound-evoked excitation and inhibition, resulting in an increase in the number of DCN neurons with larger excitatory response maps (Ma and Young, 2006; Shore et al., 2008). Changes in sounddriven and spontaneous neural activity could then lead to changes in correlated somatosensory and auditory synaptic inputs. Because neurons with primarily excitatory response maps are more likely to exhibit Hebbian-like plasticity (Koehler and Shore, 2013), a reduction in sound-driven inhibition would predict a shift toward Hebbian-like plasticity. However, this prediction is counter to the increase in antiHebbian-like plasticity tinnitus animals, suggesting that tinnitusassociated changes in bimodal plasticity are not the result of changes in the sound-driven network properties.

Alternatively, neuromodulatory systems may gate STDP (Pawlak et al., 2010). In DCN, cholinergic inputs modulate STDP at parallel fiber to fusiform cell synapses, converting it from Hebbian to anti-Hebbian (Zhao and Tzounopoulos, 2011). Increases in choline acetyltransferase activity and carbachol-induced suppression of neural activity in the DCN after noise damage (Zhang and Kaltenbach, 2000; Jin et al., 2006) suggest that increased cholinergic tone may contribute to the Hebbian to anti-Hebbian shift after noise exposure in the present study.

Somatosensory innervation of the DCN excites or inhibits principal neurons depending on whether innervation via parallel fibers is direct or through inhibitory interneurons, such as cartwheel cells (Young et al., 1995; Shore, 2005). The redistribution of unimodal somatosensory responses toward excitation in tinnitus animals suggests that new somatosensory terminals preferentially innervate fusiform cells directly versus through cartwheel cells. Together with reduced glycinergic inhibition, this would minimize the influence of cartwheel cells on bimodal stimulus timing-dependent plasticity. Parallel fiber synapses onto cartwheel cells exhibit endocannabinoid-mediated anti-Hebbian STDP that has the inverse Hebbian influence on fusiform cells (Tzounopoulos et al., 2007; Doiron et al., 2011). Thus, a reduction in cartwheel cell influence would lead to a reduction in Hebbian somatosensory influence, as observed in the present data.

After sensory deprivation, the distribution of postsynaptic NMDAR-2B changes, resulting in broader STDP timing rules in visual cortical neurons (Guo et al., 2012). The presence of
NMDAR-2B in DCN fusiform, granule, and vertical cells (Sato et al., 2000) may provide a potential mechanism for tinnitusassociated changes in STDP because synaptic plasticity is often mediated by NMDAR-initiated signaling cascades that are PKC dependent. The expression of PKC is enhanced in DCN neurons after cochlear ablation (Garcia et al., 2000), but evidence demonstrating that changes in NMDAR activity or signaling cascades are responsible for tinnitus-associated changes in STDP has not yet been provided.

\section{STDP alterations as a neural correlate for tinnitus}

Our results suggest that changes in STDP in the DCN is a new neural correlate of tinnitus. The specific STDP changes after noise exposure and tinnitus induction may drive spontaneous neural activity toward spiking patterns that represent tinnitus in DCN and higher auditory structures. Plasticity in higher auditory centers could further drive spontaneous activity toward perceptual awareness.

Bimodal plasticity that is stimulus timing-dependent has been demonstrated in auditory cortex (Basura et al., 2012) where STDP has also been implicated as a mechanism underlying stimulus timing-dependent shifts in frequency tuning (Dahmen et al., 2008). Stimulus timing-dependent frequency tuning suggests that STDP may be important for tonotopic remapping, which has been suggested as a correlate for tinnitus (Mühlnickel et al., 1998; Komiya and Eggermont, 2000). In other sensory systems, STDP is involved in elevated spontaneous activity and cortical remapping after sensory deprivation in visual (Guo et al., 2012) and somatosensory (Gambino and Holtmaat, 2012) cortices, respectively.

Our identification of tinnitus-associated changes in stimulus timing-dependent plasticity opens up avenues for novel tinnitus treatments. Already, manipulation of cortical map plasticity through vagal nerve stimulation has been proposed as a tinnitus treatment (Engineer et al., 2013). Our results suggest potential approaches to tinnitus management, including noninvasive paired stimulation of the auditory and somatosensory modalities and pharmacological targeting of STDP modulatory mechanisms.

\section{References}

Aosaki T, Miura M, Suzuki T, Nishimura K, Masuda M (2010) Acetylcholine-dopamine balance hypothesis in the striatum: an update. Geriatr Gerontol Int 10 [Suppl 1]:S148-S157.

Basura GJ, Koehler SD, Shore SE (2012) Multi-sensory integration in brainstem and auditory cortex. Brain Res 1485:95-107. CrossRef Medline

Bauer CA, Turner JG, Caspary DM, Myers KS, Brozoski TJ (2008) Tinnitus and inferior colliculus activity in chinchillas related to three distinct patterns of cochlear trauma. J Neurosci Res 86:2564-2578. CrossRef Medline

Brozoski TJ, Bauer CA, Caspary DM (2002) Elevated fusiform cell activity in the dorsal cochlear nucleus of chinchillas with psychophysical evidence of tinnitus. J Neurosci 22:2383-2390. Medline

Brozoski TJ, Wisner KW, Sybert LT, Bauer CA (2012) Bilateral dorsal cochlear nucleus lesions prevent acoustic-trauma induced tinnitus in an animal model. J Assoc Res Otolaryngol 13:55-66. CrossRef Medline

Dahmen JC, Hartley DE, King AJ (2008) Stimulus timing-dependent plasticity of cortical frequency representation. J Neurosci 28:13629-13639. CrossRef Medline

Davis KA, Miller RL, Young ED (1996) Effects of somatosensory and parallel-fiber stimulation on neurons in dorsal cochlear nucleus. J Neurophysiol 76:3012-3024. Medline

Dehmel S, Eisinger D, Shore SE (2012a) Gap prepulse inhibition and auditory brainstem-evoked potentials as objective measures for tinnitus in guinea pigs. Front Syst Neurosci 6:42. CrossRef Medline

Dehmel S, Pradhan S, Koehler S, Bledsoe S, Shore S (2012b) Noise overexposure alters long-term somatosensory-auditory processing in the dorsal cochlear nucleus: possible basis for tinnitus-related hyperactivity? J Neurosci 32:1660-1671. CrossRef Medline

Doiron B, Zhao Y, Tzounopoulos T (2011) Combined LTP and LTD of 
modulatory inputs controls neuronal processing of primary sensory inputs. J Neurosci 31:10579-10592. CrossRef Medline

Engineer ND, Møller AR, Kilgard MP (2013) Directing neural plasticity to understand and treat tinnitus. Hear Res 295:58-66. CrossRef Medline

Gambino F, Holtmaat A (2012) Spike timing-dependent potentiation of sensory surround in the somatosensory cortex is facilitated by deprivation-mediated disinhibition. Neuron 75:490-502. CrossRef Medline

Garcia MM, Edward R, Brennan GB, Harlan RE (2000) Deafferentationinduced changes in protein kinase $\mathrm{C}$ expression in the rat cochlear nucleus. Hear Res 147:113-124. CrossRef Medline

Guo Y, Huang S, de Pasquale R, McGehrin K, Lee HK, Zhao K, Kirkwood A (2012) Dark exposure extends the integration window for spike timingdependent plasticity. J Neurosci 32:15027-15035. CrossRef Medline

Haenggeli CA, Pongstaporn T, Doucet JR, Ryugo DK (2005) Projections from the spinal trigeminal nucleus to the cochlear nucleus in the rat. J Comp Neurol 484:191-205. CrossRef Medline

Heller AJ (2003) Classification and epidemiology of tinnitus. Otolaryngol Clin North Am 36:239-248. CrossRef Medline

Jin YM, Godfrey DA, Wang J, Kaltenbach JA (2006) Effects of intense tone exposure on choline acetyltransferase activity in the hamster cochlear nucleus. Hear Res 216:168-175. CrossRef Medline

Kaltenbach JA, McCaslin DL (1996) Increases in spontaneous activity in the dorsal cochlear nucleus following exposure to high intensity sound: a possible neural correlate of tinnitus. Auditory Neurosci 3:57-78.

Kaltenbach JA, Zacharek MA, Zhang J, Frederick S (2004) Activity in the dorsal cochlear nucleus of hamsters previously tested for tinnitus following intense tone exposure. Neurosci Lett 355:121-125. CrossRef Medline

Kanold PO, Young ED (2001) Proprioceptive information from the pinna provides somatosensory input to cat dorsal cochlear nucleus. J Neurosci 21:7848-7858. Medline

Kanold PO, Davis KA, Young ED (2011) Somatosensory context alters auditory responses in the cochlear nucleus. J Neurophysiol 105:1063-1070. CrossRef Medline

Koehler SD, Shore SE (2013) Stimulus timing-dependent multisensory plasticity in the guinea pig dorsal cochlear nucleus. PLoS One 8:e59828. CrossRef Medline

Koehler SD, Pradhan S, Manis PB, Shore SE (2011) Somatosensory inputs modify auditory spike timing in dorsal cochlear nucleus principal cells. Eur J Neurosci 33:409-420. CrossRef Medline

Komiya H, Eggermont JJ (2000) Spontaneous firing activity of cortical neurons in adult cats with reorganized tonotopic map following pure-tone trauma. Acta Otolaryngol 120:750-756. CrossRef Medline

Kujawa SG, Liberman MC (2009) Adding insult to injury: cochlear nerve degeneration after "temporary" noise-induced hearing loss. J Neurosci 29:14077-14085. CrossRef Medline

Levine RA (1999) Somatic (craniocervical) tinnitus and the dorsal cochlear nucleus hypothesis. Am J Otolaryngol 20:351-362. CrossRef Medline

Lindblad AC, Hagerman B, Rosenhall U (2011) Noise-induced tinnitus: a comparison between four clinical groups without apparent hearing loss. Noise Health 13:423-431. CrossRef Medline

Ma WL, Young ED (2006) Dorsal cochlear nucleus response properties following acoustic trauma: response maps and spontaneous activity. Hear Res 216:176-188. CrossRef Medline

Masquelier T, Guyonneau R, Thorpe SJ (2008) Spike timing dependent plasticity finds the start of repeating patterns in continuous spike trains. PLoS One 3:e1377. Medline

Meredith RM, Mansvelder HD (2010) STDP and mental retardation: dysregulation of dendritic excitability in fragile X syndrome. Front Synaptic Neurosci 2:10. CrossRef Medline

Mühlnickel W, Elbert T, Taub E, Flor H (1998) Reorganization of auditory cortex in tinnitus. Proc Natl Acad Sci U S A 95:10340-10343. CrossRef Medline

Pawlak V, Wickens JR, Kirkwood A, Kerr JN (2010) Timing is not everything: neuromodulation opens the STDP gate. Front Synaptic Neurosci 2:146. CrossRef Medline

Pilati N, Ison MJ, Barker M, Mulheran M, Large CH, Forsythe ID, Matthias J, Hamann M (2012) Mechanisms contributing to central excitability changes during hearing loss. Proc Natl Acad Sci U S A 109:8292-8297. CrossRef Medline

Pinchoff RJ, Burkard RF, Salvi RJ, Coad ML, Lockwood AH (1998) Modu- lation of tinnitus by voluntary jaw movements. Am J Otol 19:785-789. Medline

Roberts PD, Portfors CV (2008) Design principles of sensory processing in cerebellum-like structures: early stage processing of electrosensory and auditory objects. Biol Cybern 98:491-507. CrossRef Medline

Sanchez TG, da Silva Lima A, Brandão AL, Lorenzi MC, Bento RF (2007) Somatic modulation of tinnitus: test reliability and results after repetitive muscle contraction training. Ann Otol Rhinol Laryngol 116:30-35. Medline

Sato K, Shiraishi S, Nakagawa H, Kuriyama H, Altschuler RA (2000) Diversity and plasticity in amino acid receptor subunits in the rat auditory brain stem. Hear Res 147:137-144. CrossRef Medline

Sawtell NB (2010) Multimodal integration in granule cells as a basis for associative plasticity and sensory prediction in a cerebellum-like circuit. Neuron 66:573-584. CrossRef Medline

Shore SE (2005) Multisensory integration in the dorsal cochlear nucleus: unit responses to acoustic and trigeminal ganglion stimulation. Eur J Neurosci 21:3334-3348. CrossRef Medline

Shore SE, Koehler S, Oldakowski M, Hughes LF, Syed S (2008) Dorsal cochlear nucleus responses to somatosensory stimulation are enhanced after noise-induced hearing loss. Eur J Neurosci 27:155-168. CrossRef Medline

Suneja SK, Benson CG, Potashner SJ (1998a) Glycine receptors in adult guinea pig brain stem auditory nuclei: regulation after unilateral cochlear ablation. Exp Neurol 154:473-488. CrossRef Medline

Suneja SK, Potashner SJ, Benson CG (1998b) Plastic changes in glycine and GABA release and uptake in adult brain stem auditory nuclei after unilateral middle ear ossicle removal and cochlear ablation. Exp Neurol 151: 273-288. CrossRef Medline

Turner JG, Brozoski TJ, Bauer CA, Parrish JL, Myers K, Hughes LF, Caspary DM (2006) Gap detection deficits in rats with tinnitus: a potential novel screening tool. Behav Neurosci 120:188-195. CrossRef Medline

Turner J, Larsen D, Hughes L, Moechars D, Shore S (2012) Time course of tinnitus development following noise exposure in mice. J Neurosci Res 90:1480-1488. CrossRef Medline

Tzounopoulos T, Kim Y, Oertel D, Trussell LO (2004) Cell-specific, spike timing-dependent plasticities in the dorsal cochlear nucleus. Nat Neurosci 7:719-725. CrossRef Medline

Tzounopoulos T, Rubio ME, Keen JE, Trussell LO (2007) Coactivation of pre- and postsynaptic signaling mechanisms determines cell-specific spike timing-dependent plasticity. Neuron 54:291-301. CrossRef Medline

Wang H, Brozoski TJ, Turner JG, Ling L, Parrish JL, Hughes LF, Caspary DM (2009) Plasticity at glycinergic synapses in dorsal cochlear nucleus of rats with behavioral evidence of tinnitus. Neuroscience 164:747-759. CrossRef Medline

Young ED, Nelken I, Conley RA (1995) Somatosensory effects on neurons in dorsal cochlear nucleus. J Neurophysiol 73:743-765. Medline

Zeng C, Nannapaneni N, Zhou J, Hughes LF, Shore S (2009) Cochlear damage changes the distribution of vesicular glutamate transporters associated with auditory and nonauditory inputs to the cochlear nucleus. J Neurosci 29:4210-4217. CrossRef Medline

Zeng C, Yang Z, Shreve L, Bledsoe S, Shore S (2012) Somatosensory projections to cochlear nucleus are upregulated after unilateral deafness. J Neurosci 32:15791-15801. CrossRef Medline

Zhang J, Guan Z (2008) Modulatory effects of somatosensory electrical stimulation on neural activity of the dorsal cochlear nucleus of hamsters. J Neurosci Res 86:1178-1187. CrossRef Medline

Zhang JS, Kaltenbach JA (2000) Modulation of spontaneous activity by acetylcholine receptors in the rat dorsal cochlear nucleus in vivo. Hear Res 140:7-17. CrossRef Medline

Zhao Y, Tzounopoulos T (2011) Physiological activation of cholinergic inputs controls associative synaptic plasticity via modulation of endocannabinoid signaling. J Neurosci 31:3158-3168. CrossRef Medline

Zhou J, Shore S (2004) Projections from the trigeminal nuclear complex to the cochlear nuclei: a retrograde and anterograde tracing study in the guinea pig. J Neurosci Res 78:901-907. CrossRef Medline

Zhou J, Nannapaneni N, Shore S (2007) Vessicular glutamate transporters 1 and 2 are differentially associated with auditory nerve and spinal trigeminal inputs to the cochlear nucleus. J Comp Neurol 500:777-787. CrossRef Medline 